\title{
Advances in Janus Nanoparticles
}

\author{
Javier Reguera, Hyewon Kim, and Francesco Stellacci*
}

\begin{abstract}
The design and synthesis of Janus nanoparticles has attracted great scientific interest in recent years. Novel optical, electronic, magnetic, and superficial properties emerge from their dimensions and unique morphology at the nanoscale. Additional properties emerge from the binary spatial distribution of functionalities on a single anisotropic nanoparticle, like amphiphilicity or new catalytic effects. As a result, Janus nanoparticles are highly versatile nanomaterials with great potential in different scientific and technological fields. In this paper, some of the advances in inorganic-inorganic and inorganic-organic Janus nanoparticles are highlighted. First, the main strategies followed to synthesize Janus nanoparticles are described. Second, some of the properties and applications of Janus nanoparticles are described in four different fields: interfaces and emulsions, biomedicine, catalysis, and molecular colloids and suprastructures.
\end{abstract}

Keywords: Asymmetric particles $\cdot$ Janus nanoparticles $\cdot$ Non-centrosymmetric particles

\section{Introduction}

Janus particles are the simplest case of patchy or non-centrosymmetric particles. They are named after the Roman god Janus, who is typically represented by a double-faced head. In the same way, Janus particles have two faces with different chemical or physical properties. The large scientific effort of the last years in the creation of new synthetic methods has not only generated important contributions in a wide set of different applications such as e-ink displays, ${ }^{[1]}$ catalysis, ${ }^{[2]}$ anti-reflective coatings, ${ }^{[3]}$ or emulsifying agents, ${ }^{[4]}$ but has also improved fundamental knowledge about the assembly properties of anisotropic colloids and their use as surfactant particles. ${ }^{[5-9]}$

Several recent reviews have done an excellent job summarizing and organizing the synthesis methods and properties of the Janus particles. Walther and Müller published a systematic review on general Janus particles; ${ }^{[10]}$ Rodríguez-Fernández and Marzan edited a review on metallic particles; ${ }^{[11]}$ and Elaissari and coworkers wrote a review with a special focus on biomedical applications. ${ }^{[12]}$ The aim of this paper is not to summarize all the work that has been done on Janus particles, but to focus on the nanometer-scale Janus nanoparticles (JNPs) that are formed by inorganicinorganic or organic-inorganic materials. Some of the different strategies to synthe-

\footnotetext{
${ }^{*}$ Correspondence: Prof. F. Stellacci Institute of Materials

École Polytechnique Fédérale de Lausanne Station 12,

$\mathrm{CH}-1015$ Lausanne

Tel: + +41216937872

E-mail: francesco.stellacci@epfl.ch
}

size them, their potential applicability, and important aspects that need to be addressed in the near future will be discussed.

New properties arise in materials when the dimensions are much smaller than the macroscopic materials (bulk properties) and bigger than the molecules or atoms (molecular properties). By consensus they are defined as particles with dimensions between 1 and $100 \mathrm{~nm}$. JNPs are particles with two different sides that are also in this size range. Therefore these two parts can each possess different nanoscale properties. Among them there are optical properties, like the surface plasmon resonance of metallic NPs, sizedependent fluorescence of semiconductor quantum dots, electronic properties like those involved in surface-enhanced Raman spectroscopy (SERS), ${ }^{[13,14]}$ or the superparamagnetism of magnetic NPs. ${ }^{[15,16]}$ Particular mention is due to the high surface-to-volume ratio, yielding enhanced catalytic properties and strong interaction with biological samples, and those inherent to their asymmetry, such as the ability to stabilize interfaces or the self-assembly process on organized suprastructures. All of these exceptional qualities make JNPs excellent candidates for high value present and future applications.

Different methods to synthesize JNPs exist nowadays and are summarized below. Their development has involved a considerable challenge during the last years mainly due to the difficulties of extending colloidal Janus particles procedures to the nanometer regime. More work is still necessary in the characterization of NPs to unambiguously determine the existence and structure of JNPs, to quantify their Janus character (degree of anisotropy and polydispersity) and their yield (fraction of NPs that are Janus after a synthetic pro- cedure). Among the techniques to identify JNPs, microscopy methods such as electron microscopy and scanning probe microscopy, and macroscopic methods such as 2D NOESY NMR, contact angle, etc. are the most common. Microscopy methods can give very accurate information about the morphology but they are not useful to quantify when there is a polydisperse population of morphologies (NPs that are Janus vs. NPs that are not, or different relative sizes of the patches). On the other hand, macroscopic techniques such as contact angle or 2D NMR measure properties attributed to JNPs but they often cannot distinguish between JNPs and other kind of non-symmetric patchy NPs. There is a clear need for an improvement in the identification and quantification of JNPs in particular and patchy NPs in general, that should be solved with the combination of several techniques or with the use of new ones. Harkness et al. for example proposed one of the few examples where the degree of Janus character can be macroscopically quantified. [17] They made use of ion mobility-mass spectrometry matrix-assisted laser desorption/ionization (IM-MS MALDI) to analyze Au-thiolate complexes that protect mixed-ligand $\mathrm{Au}$ NPs. They statistically quantify the degree of phase separation base of the statistical composition of the complexes.

In the next section, the different strategies to obtain JNPs will be classified in four different approaches: methods that use masks to modify only one part of the NPs, methods based on controlled nucleation at NP surfaces, methods based on phase separation of multicomponent symmetric NPs, and methods based on self-assembled processes. Section three will be dedicated to the properties and applications of JNPs in different fields, ranging from biomedi- 
cal applications to molecular colloids. The last section will summarize and discuss some of the perspectives and challenges in the field.

\section{Synthesis Approaches}

\subsection{Surface Modification of Partially Masked Particles}

The local modification of one side of nanoparticles by the masking of the other side is the most studied method for synthesizing JNPs. It is usually done in two steps: first, isotropic NPs are placed at an interface, then a new ligand molecule, a new core material or a physical modification is introduced in a way that selectively functionalizes the exposed surface and results in JNPs.

\subsubsection{Using Flat Surfaces as Masking Interfaces}

Many flat interfaces have been used to make JNPs. Some authors use solid templates as a mask of one hemisphere of isotropic NPs and modified the exposed surface creating JNPs. Others use a liquid-air or a liquid-liquid interface and selectively functionalize NPs in one liquid phase to make JNPs.

The methods that use solid substrates as masks can be applied to a large variety of NPs. For instance, Shumaker-Parry and co-workers synthesized two different JNPs using amine-terminated glass slides and coupled them into dimers ${ }^{[18]}$ (Fig. 1). The citrate-stabilized gold NPs of $41 \mathrm{~nm}$ were immobilized on amine-terminated glass slides and then immersed in an ethanolic solution of 11-mercapto-1-undecanol $(\mathrm{MUOH})$ to replace the upper half of the ligands on the NPs. Then, the MUOH functionalized NPs were sonicated in ethanol containing either 16-mercaptoundecanoic acid or mercaptoethyl amine. The acidterminated JNPs and the amine-terminated JNPs reacted to form dimers, showing their Janus character. They also synthesized them with different sizes, and made hetero-dimers. In a different work they used the similarly developed JNPs to react with the side groups of linear polymers and achieved 1D assembly of JNPs. This was confirmed by transmission electron microscopy (TEM), and a red-shift in the plasmon resonance. ${ }^{[19]}$

Liquid-air interfaces can also be utilized to obtain JNPs. Chen and co-workers placed 1-hexanethiol (HT) coated gold NPs at the air-water interface of a Langmuir trough and made a compressed monolayer.[20] Then, they added 3-mercaptopropane-1,2-diol (MPD) in water to exchange HT to MPD. They confirmed the Janus structure by measuring contact angle of films by the Langmuir-Blodgett meth-

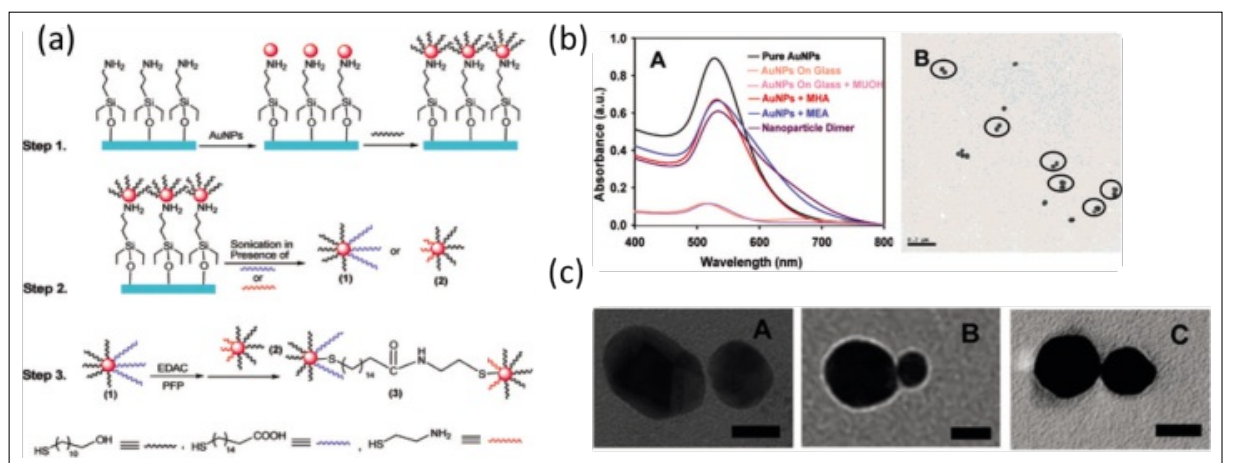

Fig. 1. a) Synthesis of two different Janus NPs using amine-functionalized glass slides. b) (c) The formation of dimers of two different Janus NPs. Reprinted with permission from ref. [18]. Copyright 2007 American Chemical Society.

od. The film made by up-stroke deposition showed a similar contact angle to that of HT-coated NP films, whereas the film made by down-stroke deposition showed a similar contact angle to that of MPDcoated NP films. As a control, the same HT NPs were exposed to a place-exchange reaction in solution. These NPs showed the contact angle between two values of allMPD coated NPs film and all-HT coated NPs film. In a further investigation, the same group studied the biphasic structure by 2D NOESY NMR. [21] They showed that the cross-peaks present in bulk-exchanged NPs disappeared for the JNPs prepared at the interface. One of the main disadvantages of the liquid-air modification is the limitation to hydrophobic-hydrophilic ligand pairs with the hydrophilic ligand being water-soluble.

Although this method was successful in the synthesis of JNPs with various sizes and different kinds of materials and functionalities, care has to be taken regarding the stability of the Janus morphology when they are out of the equilibrium situation in which they were formed. In addition, the main drawback of this method is the low-yield inherent to the limited size of the template. The yield is even lower when dealing with small JNPs, as the quantities obtained scale with the radius of the particles. To overcome this problem, alternative templates have been proposed.

\subsubsection{Using Alternative Mask Templates}

One alternative approach to overcome the low yield of the masking methods is the use of small lamellae that remain in solution and increase the amount of available surface. Li and co-workers used thiolfunctionalized polyethylene oxide (HSPEO) single crystals in order to immobilize gold NPs in solution (Fig. 2a-f). ${ }^{[22]}$ After the NPs were immobilized, the solvent-exposed ammonium groups were exchanged to thiol-functionalized ATRP initiator. Then, poly(methyl methacrylate) (PMMA) or poly(tert-butyl acrylate) (PtBA) was synthesized from the gold surface, and JNPs were obtained by dissolving the single crystal substrates. The presence of both PEO and either PMMA or PtBA was confirmed by gas permeation chromatography after iodine decomposition of the Au core. After hydrolyzing the acrylate group of PtBA, PEO/PAA-covered gold NPs were obtained, and platinum $(\mathrm{Pt})$ NPs were grown in situ on the PAA region.

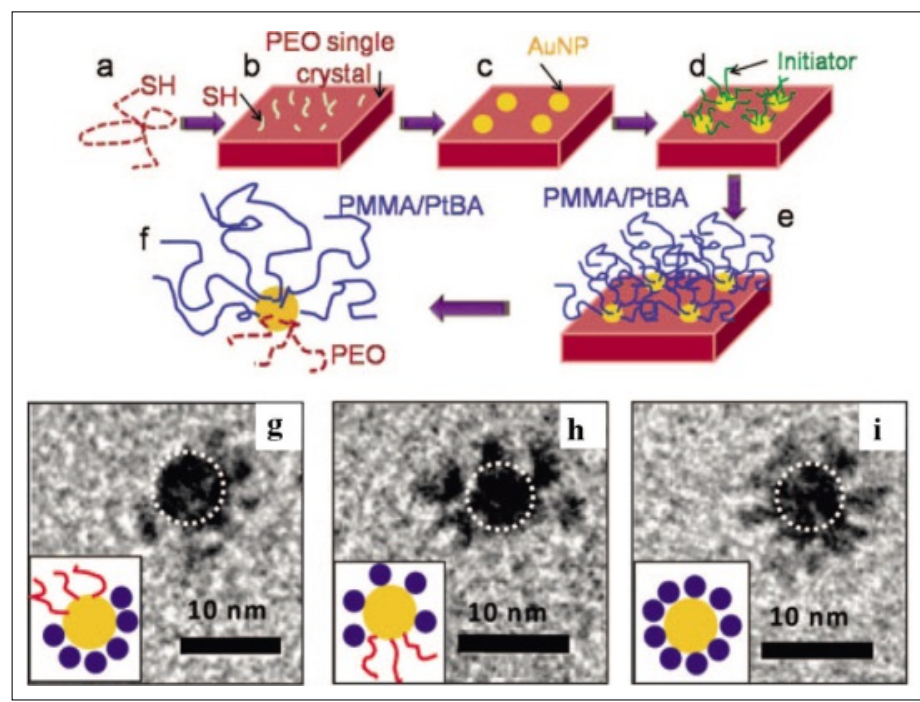

Fig. 2. a-f) Synthesis of Janus NPs using a single crystal of HS-PEO. g-i) Pt NPs grown in PAA region confirming the formation of Janus structure using this template method. Reprinted with permission from ref. [22]. Copyright 2008 American Chemical Society. 
TEM images of Pt-decorated gold NPs were taken to prove that the initial gold NPs had biphasic structure of $\mathrm{PEO} / \mathrm{PtBA}$ on the surface (Fig. 2g-i). They demonstrated that PEO/PMMA JNPs self-assemble into worm-like clusters in the mildly selective solvent (dioxane). The control sample of PEO/PMMA mixed ligand-covered NPs without Janus structure did not form such a cluster, which was also confirmed by a shift of the plasmon peak. This method can be applied to other single crystalline polymers, and a wide range of polymers can be synthesized from the surface using ATRP.

Hatton and co-workers used silica beads as a temporary support in order to synthesize $\mathrm{Fe}_{3} \mathrm{O}_{4}$ JNPs. ${ }^{[23,24]}$ They used PAA-coated $\mathrm{Fe}_{3} \mathrm{O}_{4}$ NPs to be attracted on the cationically charged silica beads. After $\mathrm{Fe}_{3} \mathrm{O}_{4}$ NPs were immobilized on the silica beads, PAA reacted with various amine-terminated polymers, resulting in magnetic JNPs. Many amine-terminated polymers could be attached on the PAAcoated $\mathrm{Fe}_{3} \mathrm{O}_{4}$ NPs producing Janus structures. They used PSSNa (pH-responsive) and PNIPAAm (temperature-responsive) for amine-terminated polymers to create stimuli-responsive magnetic NPs that the magnetic JNPs associate or dissociate depending on the applied external stimuli (Fig. 3). ${ }^{[24]}$

JNP syntheses using emulsions have also been studied. ${ }^{[25]} \mathrm{Gu}$ et al. used NPs such as $\mathrm{Au}, \mathrm{Fe}_{3} \mathrm{O}_{4}$, and $\mathrm{FePt}$, which were dissolved in organic solvent and then added to aqueous solution containing silver nitrate. The solvent mixture formed an oilin-water emulsion by ultrasonication, and one silver NP was grown onto the single precursor particles leading to dumbbell/ snowman type of JNPs. The reaction occurs rapidly and the kinetics were easily controlled in order to make various snowman-like morphologies that were observed by TEM.

Recently, Andala et al. synthesized hydrophobic/hydrophilic JNPs. ${ }^{[26]}$ They used the place-exchange reaction at a toluene-water interface. First, dodecylamine (DDA)-coated gold NPs were dissolved in the toluene phase of a toluene-water system. A toluene solution of 11-mercaptoundecanoic acid (MUA) and 1-dodecanethiol (DDT) was then added and shaken vigorously for $30 \mathrm{~min}$, after which the JNPs of DDT/MUA were formed at the interface. Interestingly the same result was obtained with a two-step ligand addition (Fig. 4). The amphiphilicity of the NPs was observed by contact angle measurement of a monolayer of NPs on hydrophilic and hydrophobic surfaces. The authors describe this process as thermodynamically driven in which the amphiphilicity of the NPs decreases the energetic configuration of the system when the NPs are at the inter-

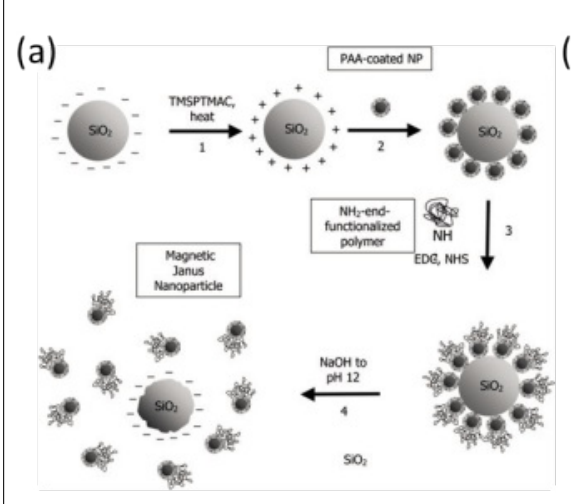

(b)

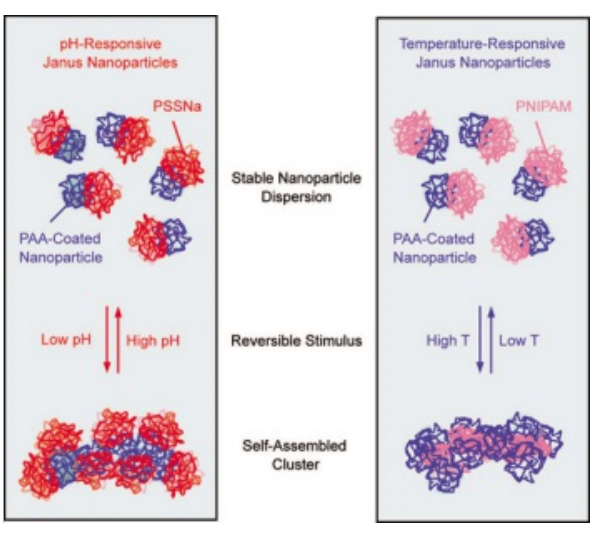

Fig. 3. a) Synthesis of magnetic Janus NPs using negatively charged $\mathrm{SiO}_{2}$ particles. b) Schematic representation of stimuli-reponsive ( $\mathrm{pH}$ and temperature) magnetic Janus NPs synthesized described in a). Reprinted with permission from refs [23] and [24]. Copyright 2012 American Chemical Society.

face in the emulsion of the two solvents. This method can be generally applied to other NPs, and the authors showed, as an example, the amphiphilicity of iron oxide magnetic JNPs.

\subsection{Phase Separation of Inorganic Materials}

JNPs can be obtained starting with symmetric NPs made of two different inorganic materials such as alloys or core-shell NPs that are then forced into phase separation through a chemical reaction of one of its components. Silica-AgI JNPs have been synthesized in this way. The process started with the synthesis of symmetric core shell $\mathrm{Ag}-\mathrm{SiO}$. Once synthesized, they were treated with $\mathrm{I}_{2}$ that was able to penetrate on the shell through the porosity of the $\mathrm{SiO}_{2}$ and react with the Ag. The AgI migrated to the exterior of the particle forming a snowman-like NP. ${ }^{[27]}$ In another example, magnetic-quantum dot JNPs of FePt-CdS were synthesized with a metastable core-shell NPs in which the CdS was in an amorphous state. A thermal treatment made the CdS crystallize and subsequently phase-separate into a JNP. ${ }^{[28]}$

\subsection{Processes Based on Controlled Surface Nucleation}

This strategy is well known to produce non-spherical inorganic-inorganic JNPs with rod-like, dumbbell-like or snowmanlike shapes. The strategy consists in using homogenous NPs in which their surface

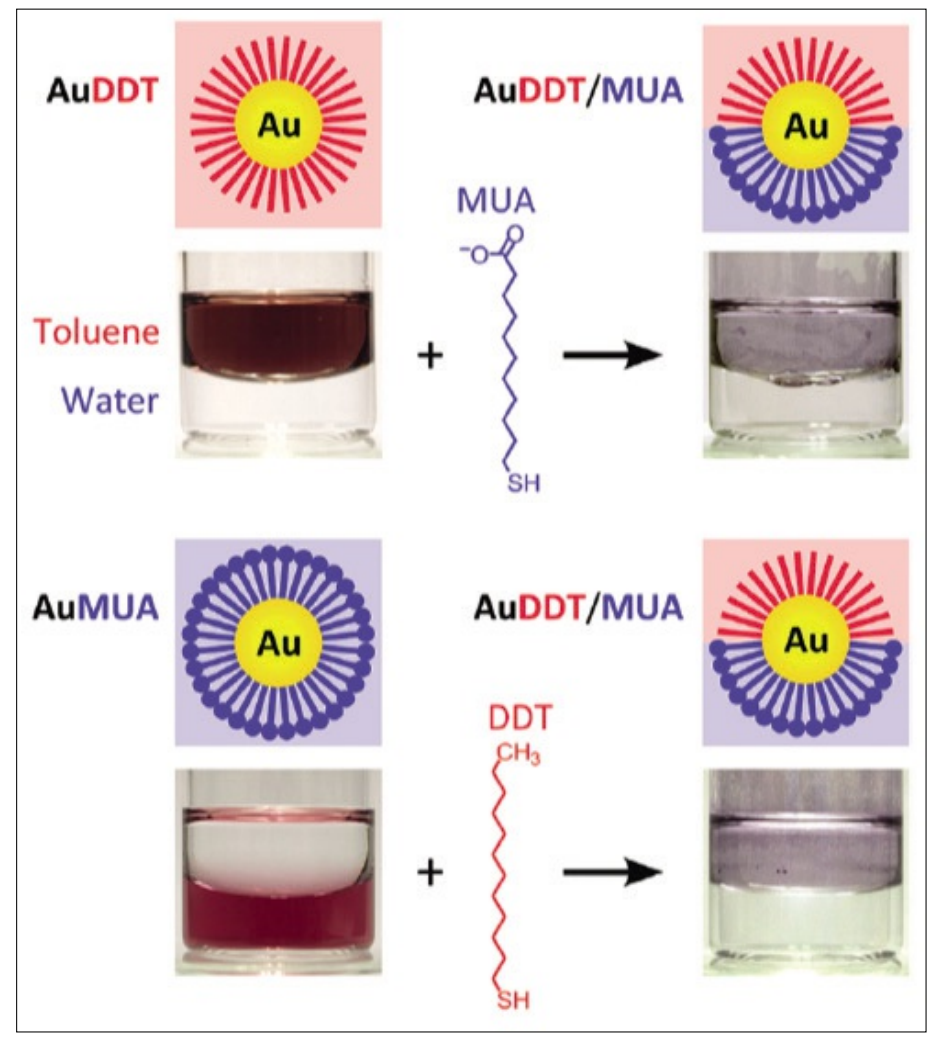

Fig. 4. The synthesis of hydrophobic/hydrophilic Janus NPs at the toluene-water interface, using selfassembly of two ligands. Reprinted with permission from ref. [26]. Copyright 2012 American Chemical Society. 
acts as a seed for the growth of a second NP of a different material. This seed and growth method has undergone extensive development and can be used nowadays with higher control on very different combinations of inorganic materials. Some of these combinations include semiconductor-metal JNPs, for instance Au NPs grown at the edge of $\mathrm{CdS}$ or $\mathrm{CdS} / \mathrm{CdSe}$ nanorods (Fig. 5):[29] a mixture of thermal- and lightinduced growth was used to achieve selective deposition of gold on one edge of the rod instead of the nanorod lateral side. Au NPs have also been grown on CdSe NPs and nanorods, ${ }^{[30]}$ and finally Co NPs have been grown on top of CdSe nano-rods. ${ }^{[31]}$ For metal-metal JNPs, different combinations like $\mathrm{Pd}-\mathrm{Au},{ }^{[32]} \mathrm{Co}-\mathrm{Au}^{[33]}$ or $\mathrm{Ni}-$ $\mathrm{Au}^{[34]}$ have been produced. Metal-metal oxide JNPs have been synthesized with $\mathrm{Co}^{-\mathrm{TiO}_{2}}{ }^{[35]}$ or $\mathrm{Au}-\mathrm{Fe}_{3} \mathrm{O}_{4} \cdot{ }^{[36]}$ In the latter case, Sun and coworkers used the decomposition of $\mathrm{Fe}(\mathrm{CO})_{5}$ in the presence of gold NPs of different sizes with subsequent surface nucleation and growth. ${ }^{[36]}$ Alternatively, they performed a direct injection of $\mathrm{HAuCl}_{4}$ into the $\mathrm{Fe}(\mathrm{CO})_{5}$ surfactant mixture, producing a two-step process of gold NP formation followed by a growth of $\mathrm{Fe}_{3} \mathrm{O}_{4}$ with the decomposition of $\mathrm{Fe}(\mathrm{CO})_{5}$.

\subsection{Self-assembly of Ligands at the Surface of an Inorganic Core}

The spontaneous phase separation of organic molecules is a highly versatile way to obtain JNPs with different functionalities. The use of block-copolymers and the selectivity of the different blocks for different solvents leading to a posterior crosslinking can be used to form different kinds of JNPs. We refer the reader to Müller's review for this subset of JNPs. ${ }^{[10]}$ The other important group of self-assembled JNPs is formed by an inorganic core and a shell composed of more than one ligand molecule. This method allows scalability, and a wide variety of combinations of ligands with different surface functionalities while keeping the optical, electric or magnetic properties of the inorganic core.

Schenning and co-workers have synthesized gold NPs covered with two different ligands with disulfides, one being hydrophobic, and the other one hydrophilic. ${ }^{[37,38]}$ They showed that in certain ligand combinations, the NPs self-assemble into disc form in water. The thickness of the disc was verified as $\sim 8-8.5 \mathrm{~nm}$, which corresponded to the bilayer of gold NPs. However, the phase separation of two ligands seemed incomplete, based on the contact angle measurement of the gold NPs films.

The synthesis of gold NPs with different binding groups has been used to create Janus Au NPs. ${ }^{[39]}$ Vilain et al. synthesized

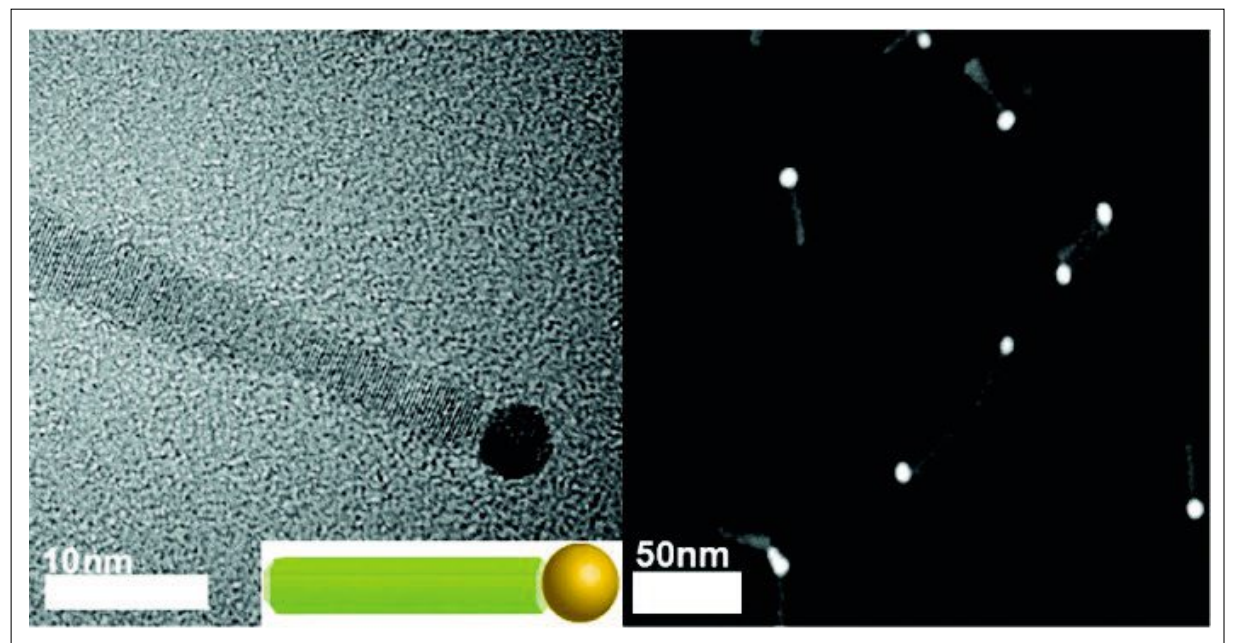

Fig. 5. Au growth on CdS nanorods, HR TEM bright field and HAADF-STEM dark field images. Reprinted with permission from ref. [29]. Copyright 2009 American Chemical Society.

Au NPs coated with diphenylphosphinine, and then performed a place-exchange reaction with various thiol ligands such as mercaptoundecanoic acid (MUA), dodecanethiol (DDT), and mercaptobenzene (MB). In order to verify the biphasic structure, UV adsorption was measured before (alldiphenylphosphinine covered NPs) and after the place-exchange reaction (combination of a thiol and diphenylphosphinine). Since the two ligands have different electronic interactions with the gold core, the electron repartition occurs on the NP shell when they are completely segregated. The experiments showed a blue-shift of UV adsorption, after ligand exchange, which indicated that the thiol and diphenylphosphinine were phase-separated. Also, they investigated DDT-exchanged NPs using FT-IR, and found homogeneous DDT domains. Finally, MUA-exchanged NPs showed different self-assembly behavior depending on the water content in THF/ water mixtures. Bilayer structures were found when the ratio between THF/water was $1 / 1$ indicating the biphasic structure of ligands on the NPs. This is a promising method to create JNPs, but the possible ligand combinations may be limited.

Harkness et al. have used IM-MS MALDI to quantify the phase-separation character of different Au NPs covered with various ligand mixtures. The clusters liberated from the NPs, that were composed of four $\mathrm{Au}$ atoms and four ligands, were analyzed and compared with the expected binomial distribution. The results show several degrees of phase separation where the mixtures of Tiopronin (Tio) and mercaptoundecyltetraethylene glycol (MUTEG) were the ones showing higher degree of phase separation. ${ }^{[17]}$

Our group has synthesized JNPs with various combinations of different thiolated ligands. The Janus morphology is obtained by the right balance of enthalpy and entropy of mixing. Enthalpically, two ligands tend to phase separate and minimize the number of interfaces between two ligands. However, entropically there is a tendency of the ligands to mix. When two ligands with different size are close together, conformational entropy of the extra length of the longer molecule plays an important role and gives rise to phaseseparation in narrow domains (Fig. 6a). ${ }^{[40]}$ On the other hand, it has been theoretically predicted that when the size of the gold core becomes smaller, ${ }^{[41]}$ the interfacial entropy contribution becomes less and less relevant since the available space and mobility of the long ligands is already high. Under this condition, the final morphology is primarily determined by the enthalpy of separation; hence, for small particles, two ligands phase-separate into two bulk phases, resulting in JNPs (Fig. 6b). Our group has synthesized gold NPs with several sizes and various combinations of ligands. Different characterization methods have demonstrated a complete phase separation in Janus morphology for small NP

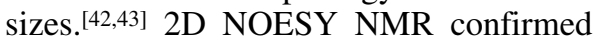
the formation of JNPs for small NPs sizes with ligands 1,1',4',1'"-terphenyl-4-thiol (TPT) and 1-tetradecanethiol (TDT). ${ }^{[42]}$ The phase separation was identified by the absence of cross peaks in the NOESY spectrum. 2D NOESY NMR was also used, together with the variation of chemical shifts in $1 \mathrm{D}^{1} \mathrm{H}$ NMR at different ligand ratios and STM, to determine the Janus character of diphenyl-4-thiol (DPT) and 1-dodecanethiol (DDT). ${ }^{[43]}$ In addition, the formation of Janus morphology was analyzed after chemical linkage by means of different methods such as TEM and analytical ultracentrifugation (AUC). ${ }^{[42]}$ Janus NPs were identified when the diameter of the metal cores was smaller than $1.5 \mathrm{~nm}$. Mixed morphologies of Janus and striped NPs are predicted to exist when 
the gold core size is between 1.5-3 nm. However, it should be noted that this range is not absolute and it will critically depend on the nature of the ligand mixture used. For example, for MUA, 1-octanethiol (OT) JNPs were found for sizes around 3 $\mathrm{nm}$, and also Janus morphology has been found in bigger NPs when they are coated with a 1-mercaptoundecanol (MUOH) and 1-mercaptobutanol $(\mathrm{MBOH})$ mixture. This indicates that other phase separation mechanisms may also be involved. ${ }^{[44]}$

\section{Properties and Applications}

There are multiple fields where JNPs have already been successfully used and several others in which JNPs are expected to cause a fundamental improvement. The wide variety of applications of these NPs is mainly due to the large number of combinations of materials and shapes that comprise the JNP zoo. Here we show a few of these applications in fields where JNPs have contributed to create a considerable advance:

\subsection{Stabilization of Interfaces}

Higher absorption at interfaces is maybe one of the more straightforward properties of amphiphilic JNPs. They combine the Pickering effect (NPs adsorbed at liquid-liquid interfaces) with the surfactantlike behavior of JNPs. Binks et al. ${ }^{[45]}$ show theoretically that JNPs are adsorbed more strongly at interfaces than homogenous NPs. Fig. 7 shows for example the desorption energy of $10 \mathrm{~nm}$ JNPs at several degrees of contact angle difference between the two parts of JNPs. The adsorption energy increases several times and makes them more stable at average contact angles different from $90^{\circ}$. This result has also

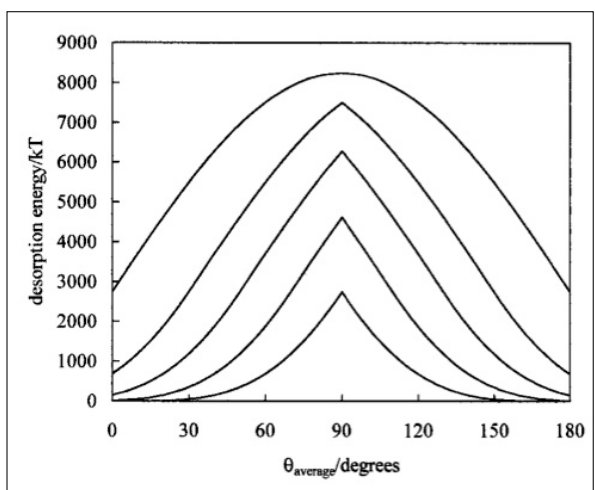

Fig. 7. Variation of particle desorption energy with area-weighted average contact angle for particles of radius $10 \mathrm{~nm}$ and $R=90^{\circ}$. The oilwater tension was set to $36 \mathrm{mN} \mathrm{m}^{-1}$. In order of increasing desorption energies, the curves refer to $\Delta \theta$ of 0 (the homogeneous particle case), $20,40,60$, and $90^{\circ}$. Reprinted with permission from ref. [45]. Copyright 2011 American Chemical Society. been experimentally proved with $\mathrm{Fe}_{3} \mathrm{O}_{4}-$ Au JNPs compared with the respective homogenous NPs using pendant drop contact angle measurements on water/hexane (Fig. 8). ${ }^{[4]}$

As the adsorption energy strongly depends on the size of the particles, the Janus character can enable small NPs to go to interfaces and be used to stabilize emulsions. In addition it has been shown that JNPs are more effective to inhibit the coalescence of the dispersed phase on the Pickering emulsions, which increases their long applicability range. ${ }^{[46]}$ The use of JNPs instead of big particles could then form emulsions of smaller droplet size and with less porosity. Additionally, the two faces of the JNPs situated at the different liquid interfaces can be used for various purposes taking advantage of their different optical, electric or magnetic properties. Crossley et al. used JNPs of iron oxide with attached nanotubes to stabilize a water-in-oil emulsion that, with the addition of palladium NPs, was able to catalyze biofuel reactions. ${ }^{[47]}$ The products of the reaction were able to migrate from one phase to the other through the porosity of the interphase (Fig. 9). ${ }^{[4,48]}$ Together with adsorption at liquid-liquid interfaces, JNPs can also be used to adhere at liquidsolid interfaces or solid-solid interfaces such as the boundaries of block-copolymer domains. ${ }^{[49]}$

\subsection{Bioapplications}

Although several applications of JNPs have appeared in the last year in the biomedical field, nanobiomedicine is still one of the fields where JNPs will clearly play a fundamental role in the near future. The dimensions of JNPs with controllable, separated functionalities are on the order of biomolecule dimensions, making them excellent candidates for interactions with

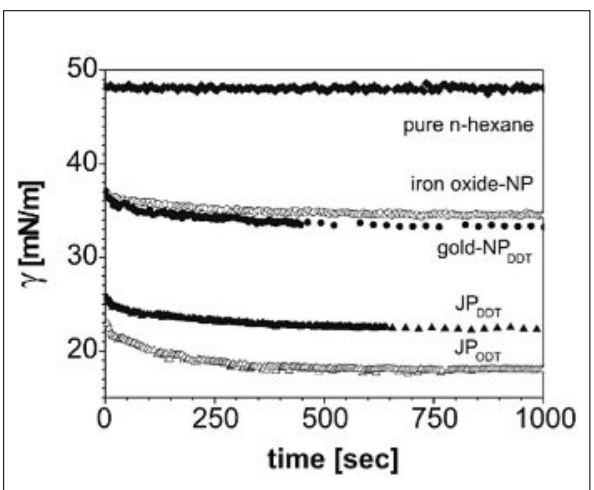

Fig. 8. Interfacial tension vs time. Water was used as the drop phase, and n-hexane was used as the ambient phase in which the nanoparticles were diluted. (NP: homogeneous nanoparticles, JP: Janus particles). The gold moieties were modified using dodecanethiol (DDT) or octadecanethiol (ODT).) Reprinted with permission from ref. [4]. Copyright 2006 American Chemical Society.

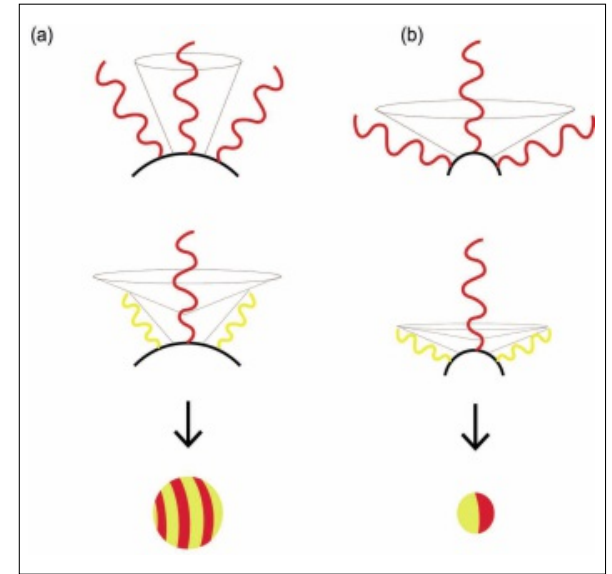

Fig. 6. Schematic drawing illustrating the conformational entropy for ligands on NPs. Schematic self-assembly morphology and predicted mechanisms. For relatively larger radii of curvature (a) the presence of shorter ligands allows longer ones to have a larger free volume; and hence, gain conformational entropy. One can divide the cone that shows a free volume of a long ligand in two parts: one whose angle is determined by the packing of the molecules and the other due to this interfacial free volume. (b) As the radius of curvature decreases, the first cone becomes the dominant, and makes the second one irrelevant. Reprinted with permission from ref. [42]. Copyright 2012 John Wiley and Sons.

different molecules and systems of the body. Additionally they can combine different functionalities such as targeted drug delivery systems, biomolecular biosensing or molecular imaging, all of them of extreme importance in the biomedical field.

JNPs can be used to transport molecules with different biofunctionalities. For example protein cages coupled with streptavidin have been engineered as JNPs by partially masked modification. These JNPs combine the transport capabilities of the protein cages (nanoplatforms composed of monomeric protein subunits that have been engineered to transport and encapsulate therapeutic agents) with a streptavidin molecule that acts as an anchoring point for many biotinylated molecules such as those that bind to cellular receptors or monoclonal antibodies that target microbial pathogens. ${ }^{[50]}$

Application of JNPs allows, in an easy manner, the use of one side dedicated to target-specific receptors while the other is dedicated to sensing events. Wu et al. have synthesized nanocoral JNPs (Fig. 10) of polystyrene (PS) and Au, where the PS part is functionalized with target recognition molecules while the Au part is dedicated to SERS detection. [51] More generally, the enhancement of physical properties of inorganic NPs can be used for all kinds of sensing events that use optical, magnetic or electronic properties. Dumbbell-like NPs 
of FePt-Au having polyethylene glycolbased ligands on their surfaces have been used as probes for magnetic resonance imaging (MRI) of tumor cell targets. [52]

Moreover, JNPs can be used as antimicrobial agents that replace conventional antibiotics by the antibacterial effect of Ag. Zhang et al. have created Janus nanorods (JNRs) composed of a magnetic head and a silica rod functionalized with Ag NPs and positively charged quaternary amines (Fig. 11). ${ }^{[53]}$ The JNRs were able to attach to $E$. coli bacteria, and they were afterwards collected using magnetic fields. At the same time the Ag NPs inhibited the growing of bacteria colonies.

Apart from typical biomedicine applications such as biosensing or drug delivery systems, the use of JNPs can be easily extended to very diverse biomaterial applications changing the way medicine is applied, from surface-modified scaffolds to beta amyloid formations in brain diseases and to virus detection. Certainly the field of nanobiomedicine is still developing and its improvement will depend on the formation of new nanostructures with spatially controlled functionalities and where the new JNPs will play an important and necessary role.

\subsection{Catalysis}

Inorganic NPs of metals and metal oxides have shown catalytic properties in a vast number of reactions that make use of the high surface to volume ratio of the NPs. This catalytic activity can nevertheless be enhanced with the right combination of materials on JNPs. Au-TiO 2 JNPs are a good example of this combined effect. $\mathrm{Au}-\mathrm{TiO}_{2}$ dimer NPs have shown photocatalytic activity in the oxidative conversion of methanol into formaldehyde. ${ }^{[53,54]}$ The enhanced photocatalytic activity compared to simple $\mathrm{TiO}_{2}$ NPs was due to the charge separation of photogenerated electrons and holes at the $\mathrm{Au}-\mathrm{TiO}_{2}$ interface helped by the close proximity of the gold NP. Au$\mathrm{TiO}_{2}$ particles have shown also photocatalytic properties for $\mathrm{H}_{2}$ generation from an isopropanol aqueous solution under visible light that highly enhanced the catalytic activity with respect to the corresponding homogenous NPs (Fig. 12). ${ }^{[55]}$ With a different combination of materials, Wang et al. used $\mathrm{Au}-\mathrm{Fe}_{3} \mathrm{O}_{4}$ and $\mathrm{Pt}-\mathrm{Fe}_{3} \mathrm{O}_{4}$ JNPs for the oxidation of $\mathrm{CO} .{ }^{[56]}$

In a different kind of application, nanomotors and nanojets can also make use of the asymmetric catalytic properties of the JNPs. Mallouk and coworkers have shown how $\mathrm{Pt}-\mathrm{Au}$ JNRs can move, driven by the catalytic effect on the decomposition of $\mathrm{H}_{2} \mathrm{O}_{2}$. ${ }^{[5]}$ The movement of the nanorods can be directed with the use of an external field, for example with the inclusion of magnetic patches. ${ }^{[58]}$

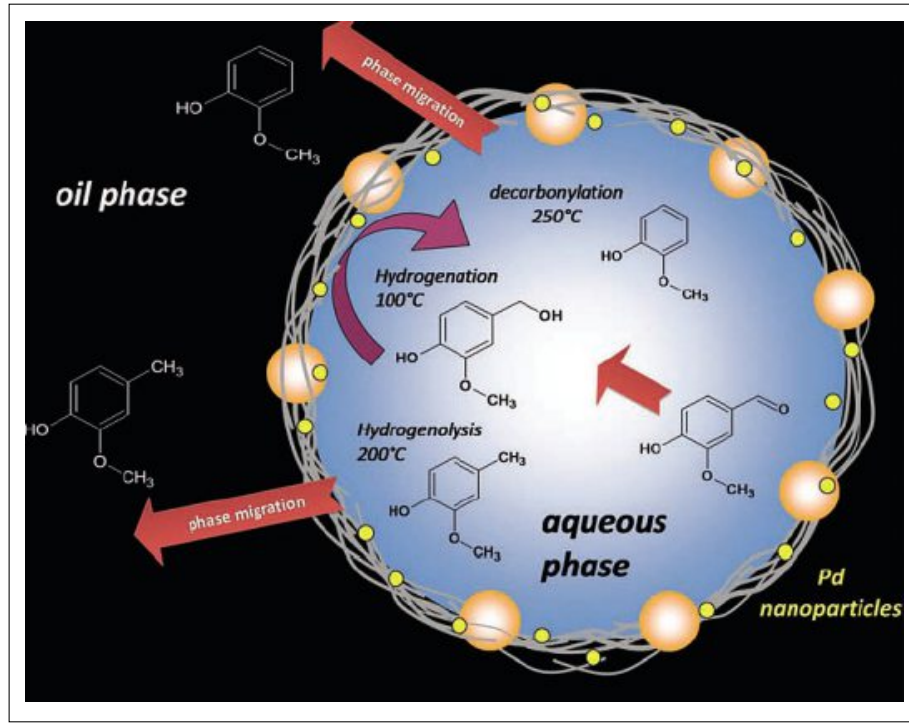

Fig. 9. Schematic illustration of the reactions taking place at the water/oil interface in the solid-stabilized emulsions. Depending on the reaction temperature, the prevailing reactions are hydrogenation, hydrogenolysis, or decarbonylation, and depending on the relative solubilities, the products remain in the aqueous phase or migrate to the oil phase. Reprinted with permission from ref. [47]. Copyright 2010 AAAS.

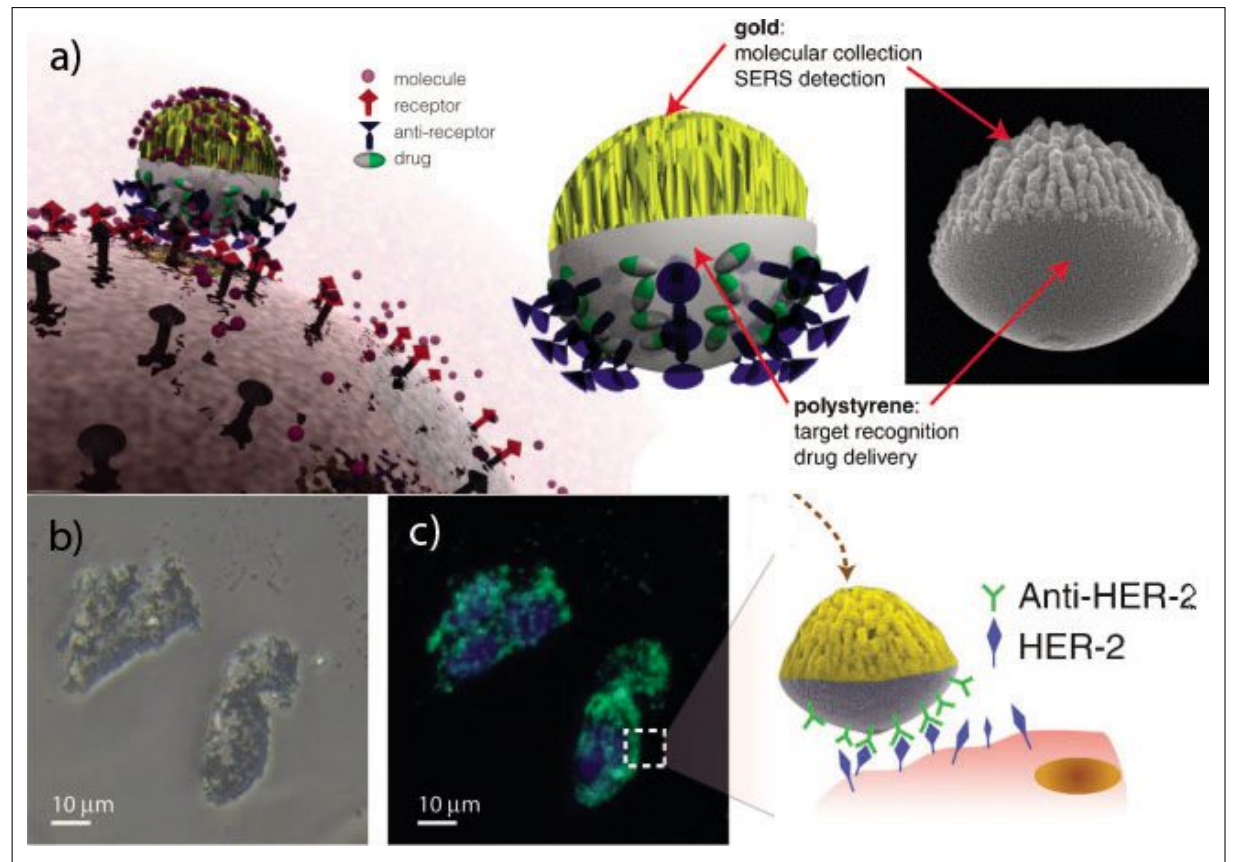

Fig. 10. a) Schematic illustration of nanocorals as multifunctional Janus nanoprobes for targeting and sensing; b) and c) nanocorals attached to BT474 cell surfaces with HER-2/anti-HER-2 binding. Reproduced with permission from ref. [51]. Copyright 2010 John Wiley and Sons.

\subsection{Higher-order Structures}

Likewise, reducing the dimensions of the material to the nanometer regime to produce nano-objects has brought quite unique properties, the arrangement of the produced nano-objects in higher order suprastructures can bring new properties arising by the collaborative effect of the nano-objects. These properties have been explored for spherical colloids, where due to the high symmetry, only closed packed structures can form (fcc and hcp lattices). Glotzer et al. have reviewed the different anisotropic NPs produced nowadays, and discussed how an increase in anisotropy can lead to a large number of new suprastructures whose function is still to be explored. ${ }^{[59]}$
Apart from new supracrystals with different lattices groups or JNPs perpendicularly organized at interfaces, the assembly of few JNPs in solution can give rise to interesting structures and molecular colloids. Granick and co-workers have explored, theoretically and with the use of bicompartmental Janus colloids, the assembly of structures of JNPs from small clusters to triple helices with at most six neighbor particles. ${ }^{[60]}$ Pozzo and coworkers have shown the formation of small clusters structures from 2 to 5 NPs formed with a mixture of PEG and butanethiol. They attributed those structures to Janus formation due to the phase separation of the ligands. ${ }^{[61]} \mathrm{Li}$ and coworkers synthesized Au JNPs covered with PEO and PMMA through a masked 


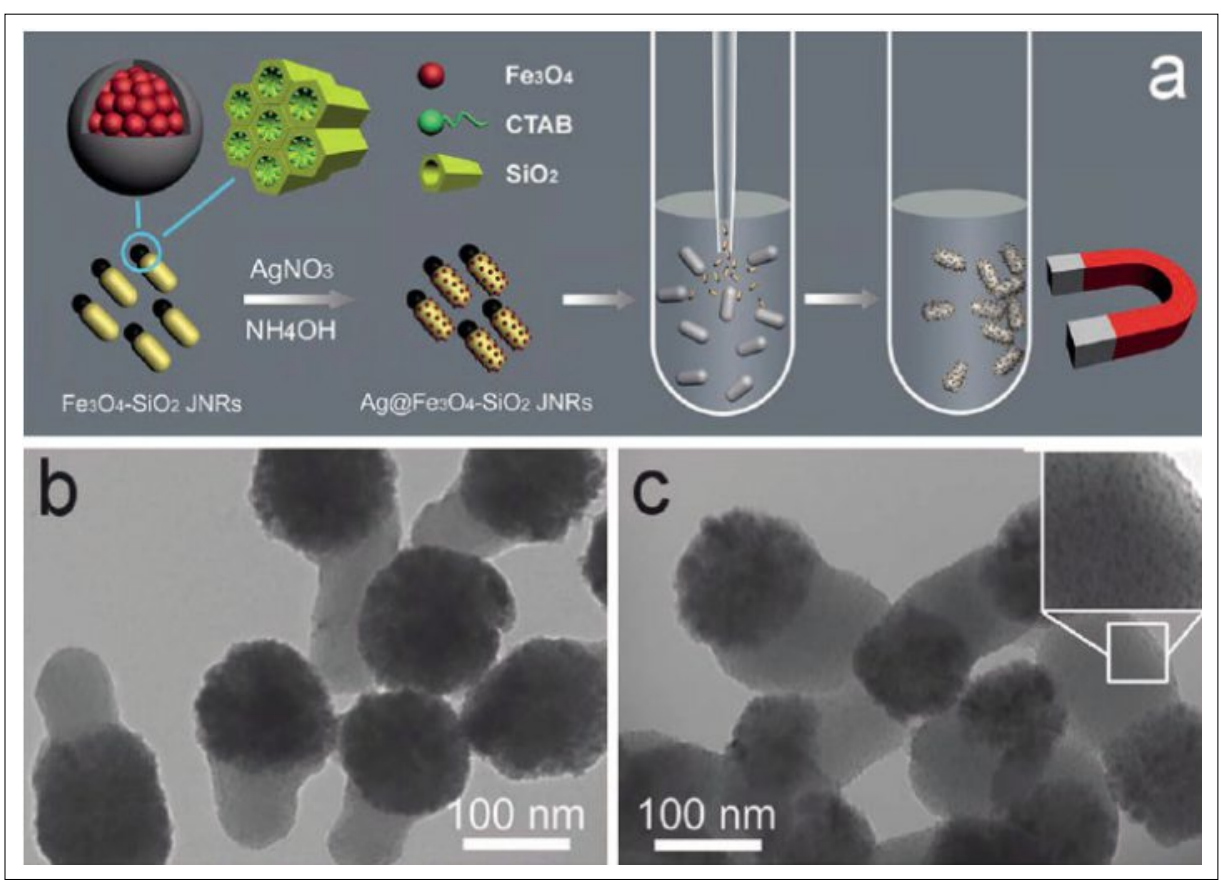

Fig. 11. a) Schematic illustration of a typical procedure for the in situ synthesis of AgNPs@ $\mathrm{Fe}_{3} \mathrm{O}_{4}-\mathrm{SiO}_{2}$ JNRs and their sterilization and separation process. b) and c) TEM images of AgNPs@ $\mathrm{Fe}_{3} \mathrm{O} 4-\mathrm{SiO}_{2}$ JNRs with different lengths. Reprinted with permission from ref. [53]. Copyright 2010 RSC.

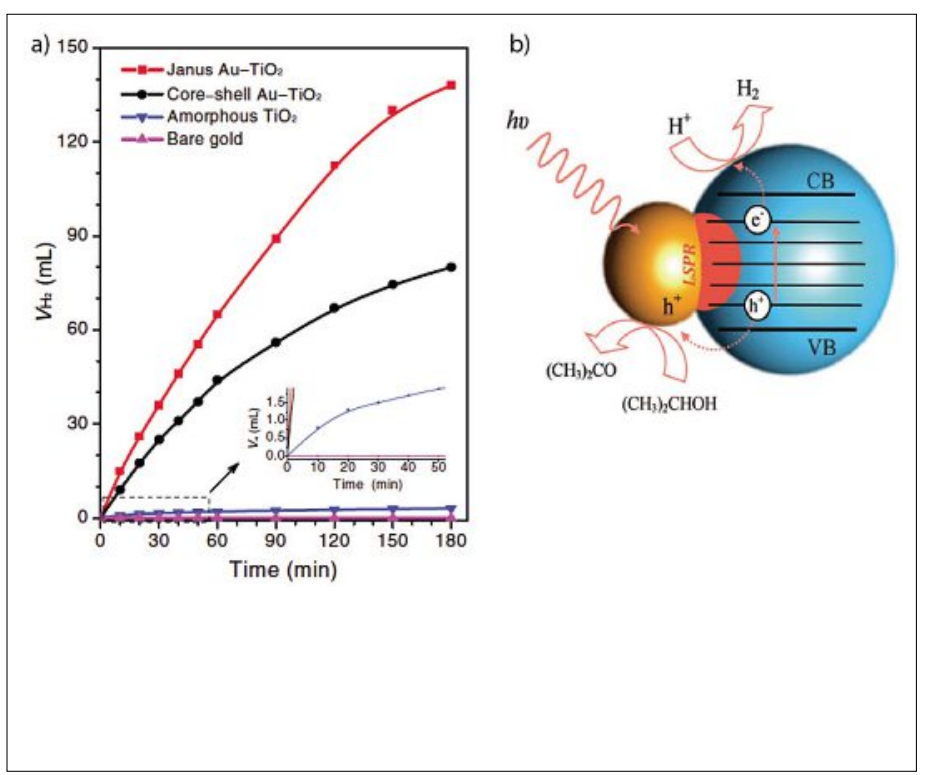

Fig. 12. a) $\mathrm{H}_{2}$ generated under visible light irradiation from a halogen lamp using Janus and core-shell Au $50 \mathrm{~nm} \mathrm{TiO}_{2}$ nanostructures, as well as amorphous $\mathrm{TiO}_{2}$ and bare gold nanoparticles (50 nm). b) Proposed photocatalytic process for efficient hydrogen generation using the Janus Au$\mathrm{TiO}_{2}$ nanostructures, based on excitation of the LSPR under visible-light irradiation. Reproduced with permission from ref. [55]. Copyright 2010 John Wiley and Sons.

process. ${ }^{[62,63]}$ The self-assembly of these PEO-PMMA JNPs in clusters was driven by the exposition of the JNPs to different solvents and by exploiting the difference of solvophobicity of the two different polymers.

\section{Summary and Outlook}

Different key aspects of JNPs have been described here. The increasing set of diverse strategies to synthesize JNPs allows one to choose the most suitable according to the demands of an application in terms of materials, amount needed, NP sizes, and functionalities. The develop- these difficulties, like the use of emulsions or small lamellae that allow the reactions to be undertaken in bulk solution. The methods based on controlled surface nucleation and the phase separation of inorganic materials can produce larger amounts, but with less control of the JNPs polydispersity than the mask-based methods. They have been shown to be very useful synthetic methods for inorganic-inorganic NPs, especially for hybrid metal-metal oxide NPs. Finally, self-assembled processes have a huge potential due to the high yield of the bulk synthesis together with the big variety of functionalities that organic synthesis can provide. The challenge in this last case is either to find the right molecules, from the existing ones, that self-assemble in a phase-separated manner, or to find the design rules through the macromolecular chemistry that will give rise to Janus systems.

Special care has to be taken when samples are prepared in conditions that are not in equilibrium, as they could evolve in the medium of the corresponding application. For example, in the case of metallic NPs covered with thiolate ligands, it has been proven that these ligands can be easily replaced when they are surrounded by an excess of other thiolate ligands. ${ }^{[64]}$ In addition, these ligands have been shown to move laterally on the metallic flat surfaces ${ }^{[65]}$ and on NPs, although the time scale of this movement still remains controversial and is probably strongly dependent on the packing of the organic layer and on the defects in the underlying gold surface. ${ }^{[66]}$ In any case, the non-equilibrium situation has to be addressed in the near future to improve the quality of the JNPs in different applications. Three possible solutions can be used to address this problem. The first one would be to establish the time scale in which the JNPs would reach equilibrium (the kinetics of mixing) and to reduce the applicability of the JNPs to that time scale. The second would be to immobilize the ligands once they are in the Janus configuration. Song et al. performed a cross-linking of one of the ligands through diacetylene homocoupling after a partially-masked synthesis method, that inhibits the possible diffusion of the ligands. ${ }^{[67]}$ The third possibility is to find configurations that are truly in equilibrium through the election of the right ligand molecules, NPs sizes, and the media in which they are used.

There are numerous applications of JNPs and they are increasing as the yield and control of synthetic methods improves. The spatial control of properties such as amphiphilicity makes them excellent candidates for interface-related applications such as the stabilization of emulsions through porous membranes. The biomedical field is expected to be one where more 
new applications will be developed as the field of nanobiotechnology is experiencing a technological revolution itself. In the field of catalysis we have learnt during these past years that we can amplify the catalytic activity not only with the sum of the properties of two materials that are in the same NP but also through new effects born through the synergy of the two parts. $\mathrm{Au}-\mathrm{TiO}_{2}$ JNPs are probably the first example of this process but surely new combinations of metal-metal oxide JNPs will show new combined catalytic activities. Finally, manipulation of hierarchic structures formed by the self-assembly of selfassembled structures at different length scales is also fundamental and anisotropic nano-objects such as JNPs are playing an important role in the manipulation of matter through bottom-up approaches. Big advances have been achieved recently in the formation of molecular colloids of JNPs. The improvements in the comprehension of these new structures will give rise to new physicochemical properties and certainly will lead to new interesting applications.

On the whole, new revolutionary applications are emerging through the use of asymmetric NPs, especially Janus particles. Several methods currently exist to produce JNPs, but much work is required to create new methods that are able to produce high quantities in a precise and reproducible manner, compatible with the diverse array of materials and functionalities that chemistry and material science has to offer. Also, binary NP characterization must be improved for the unambiguous identification of Janus NPs and to give information about their polydispersity and their quality.

Received: July 4, 2013

[1] T. Nisisako, T. Torii, T. Takahashi, Y. Takizawa, Adv. Mater. 2006, 18, 1152.

[2] D. J. Cole-Hamilton, Science 2010, 327, 41.

[3] H. Y. Koo, D. K. Yi, S. J. Yoo, D. Y. Kim, $A d v$. Mater. 2004, 16, 274.

[4] N. Glaser, D. J. Adams, A. Boker, G. Krausch, Langmuir 2006, 22, 5227.

[5] S. Gangwal, O. J. Cayre, O. D. Velev, Langmuir 2008, 24, 13312.

[6] L. Hong, A. Cacciuto, E. Luijten, S. Granick, Nano Lett. 2006, 6, 2510.

[7] L. Hong, A. Cacciuto, E. Luijten, S. Granick, Langmuir 2008, 24, 621 .

[8] F. Sciortino, A. Giacometti, G. Pastore, Phys. Rev. Lett. 2009, 103, 237801.
[9] A. Goyal, C. K. Hall, O. D. Velev, Phys. Rev. E 2008, 77, 031401.

[10] A. Walther, A. H. Müller, Chem. Rev. 2013, 113, 5194.

[11] D. Rodriguez-Fernandez, L. M. Liz-Marzan, Part. Part. Syst. Charact. 2013, 30, 46.

[12] C. Kaewsaneha, P. Tangboriboonrat, D. Polpanich, M. Eissa, A. Elaissari, ACS Appl. Mater. Interfaces 2013, 5, 1857.

[13] A. J. Wilson, K. A. Willets, WIREs Nanomed. Nanobiotechnol. 2013, 5, 180.

[14] D. Cialla, A. Marz, R. Bohme, F. Theil, K. Weber, M. Schmitt, J. Popp, Anal. Bioanal. Chem. 2012, 403, 27.

[15] S. Bedanta, W. Kleemann, J. Phys. D: Appl. Phys. 2009, 42, 013001

[16] U. Jeong, X. W. Teng, Y. Wang, H. Yang, Y. N. Xia, Adv. Mater. 2007, 19, 33.

[17] K. M. Harkness, A. Balinski, J. A. McLean, D. E. Cliffel, Angew. Chem. Int. Ed. 2011, 50, 10554.

[18] R. Sardar, T. B. Heap, J. S. Shumaker-Parry, J. Am. Chem. Soc. 2007, 129, 5356.

[19] R. Sardar, J. Shumaker-Parry, Nano Lett. 2008, 8,731 .

[20] S. Pradhan, L. P. Xu, S. W. Chen, Adv. Funct. Mater. 2007, 17, 2385.

[21] S. Pradhan, L. E. Brown, J. P. Konopelski, S. W. Chen, J. Nanopart. Res. 2009, 11, 1895.

[22] B. Wang, B. Li, B. Zhao, C. Y. Li, J. Am. Chem. Soc. 2008, 130, 11594.

[23] M. Lattuada, T. A. Hatton, J. Am. Chem. Soc. 2007, 129, 12878.

[24] T. Isojima, M. Lattuada, J. B. Vander Sande, T. A. Hatton, ACS Nano 2008, 2, 1799.

[25] H. Gu, Z. Yang, J. Gao, C. K. Chang, B. Xu, J. Am. Chem. Soc. 2005, 127, 34 .

[26] D. Andala, S. Shin, H. Lee, K. Bishop, ACS Nano 2012, 6, 1044.

[27] M. Giersig, T. Ung, L. M. Liz-Marzan, P. Mulvaney, Adv. Mater. 1997, 9, 570.

[28] H. W. Gu, R. K. Zheng, X. X. Zhang, B. Xu, J. Am. Chem. Soc. 2004, 126, 5664.

[29] G. Menagen, J. E. Macdonald, Y. Shemesh, I. Popov, U. Banin, J. Am. Chem. Soc. 2009, 131, 17406.

[30] T. Mokari, C. G. Sztrum, A. Salant, E. Rabani, U. Banin, Nature Mater. 2005, 4, 855 .

[31] J. Maynadie, A. Salant, A. Falqui, M. Respaud, E. Shaviv, U. Banin, K. Soulantica, B. Chaudret, Angew. Chem. Int. Ed. 2009, 48, 1814.

[32] P. H. C. Camargo, Y. Xiong, L. Ji, J. M. Zuo, Y. N. Xia, J. Am. Chem. Soc. 2007, 129, 15452.

[33] T. Wetz, K. Soulantica, A. Talqui, M. Respaud, E. Snoeck, B. Chaudret, Angew. Chem. Int. Ed. 2007, 46, 7079.

[34] L. O. Mair, B. Evans, A. R. Hall, J. Carpenter, A. Shields, K. Ford, M. Millard, R. Superfine, $J$. Phys. D: Appl. Phys. 2011, 44, 125001.

[35] M. Casavola, V. Grillo, E. Carlino, C. Giannini, F. Gozzo, E. F. Pinel, M. A. Garcia, L. Manna, R. Cingolani, P. D. Cozzoli, Nano Lett. 2007, 7, 1386.

[36] H. Yu, M. Chen, P. M. Rice, S. X. Wang, R. L. White, S. Sun, Nano Lett. 2005, 5, 379.

[37] R. T. M. Jakobs, J. van Herrikhuyzen, J. C. Gielen, P. C. M. Christianen, S. C. J. Meskers, A. P. H. J. Schenning, J. Mater. Chem. 2008, 18, 3438.
[38] J. van Herrikhuyzen, G. Portale, J. C. Gielen, P. C. M. Christianen, N. A. J. M. Sommerdijk, S. C. J. Meskers, A. P. H. J. Schenning, Chem. Commun. 2008, 697.

[39] C. Vilain, F. Goettmann, A. Moores, P. Le Floch, C. Sanchez, J. Mater. Chem. 2007, 17, 3509.

[40] A. M. Jackson, Y. Hu, P. J. Silva, F. Stellacci, J. Am. Chem. Soc. 2006, 128, 11135.

[41] C. Singh, P. K. Ghorai, M. A. Horsch, A. M. Jackson, R. G. Larson, F. Stellacci, S. C. Glotzer, Phys. Rev. Lett. 2007, 99, 226106.

[42] H. Kim, R. P. Carney, J. Reguera, Q. K. Ong, X. Liu, F. Stellacci, Adv. Mater. 2012, 24, 3857.

[43] X. Liu, M. Yu, H. Kim, M. Mameli, F. Stellacci, Nat. Commun. 2012, 3, 1182 .

[44] Q. K. Ong, J. Reguera, P. J. Silva, M. Moglianetti, K. Harkness, M. Longobardi, K. Mali, Christoph Renner, S. D. Feyter, F. Stellacci, ACS Nano 2013, DOI: 10.1021/ nn402414b.

[45] B. P. Binks, P. D. I. Fletcher, Langmuir 2001, 17, 4708.

[46] H. Fan, A. Striolo, Soft Matter 2012, 8, 9533.

[47] S. Crossley, J. Faria, M. Shen, D. E. Resasco, Science 2010, 327, 68.

[48] D. J. Cole-Hamilton, Science 2010, 327, 41.

[49] L. Q. Wang, J. P. Lin, X. M. Zhu, RSC Advances 2012, 2, 12870 .

[50] P. A. Suci, S. Kang, M. Young, T. Douglas, J. Am. Chem. Soc. 2009, 131, 9164.

[51] L. Y. Wu, B. M. Ross, S. Hong, L. P. Lee, Small 2010, 6, 503.

[52] J. S. Choi, Y. W. Jun, S. I. Yeon, H. C. Kim, J. S. Shin, J. Cheon, J. Am. Chem. Soc. 2006, 128, 15982.

[53] L. Zhang, Q. Luo, F. Zhang, D. M. Zhang, Y. S. Wang, Y. L. Sun, W. F. Dong, J. Q. Liu, Q. S. Huo, H. B. Sun, J. Mater. Chem. 2012, 22, 23741.

[54] S. Pradhan, D. Ghosh, S. Chen, ACS Appl. Mater. Interfaces 2009, 1, 2060.

[55] Z. W. Seh, S. Liu, M. Low, S. Y. Zhang, Z. Liu, A. Mlayah, M. Y. Han, Adv. Mater. 2012, 24, 2310.

[56] C. Wang, H. F. Yin, S. Dai, S. H. Sun, Chem. Mater. 2010, 22, 3277.

[57] Y. Wang, R. M. Hernandez, D. J. Bartlett, J. M. Bingham, T. R. Kline, A. Sen, T. E. Mallouk, Langmuir 2006, 22, 10451.

[58] T. R. Kline, W. F. Paxton, T. E. Mallouk, A. Sen, Angew. Chem. Int. Ed. 2005, 44, 744.

[59] S. C. Glotzer, M. J. Solomon, Nature Mater. 2007, 6, 557

[60] Q. Chen, J. K. Whitmer, S. Jiang, S. C. Bae, E. Luijten, S. Granick, Science 2011, 331, 199.

[61] K. Larson-Smith, D. C. Pozzo, Soft Matter 2011, 7, 5339.

[62] B. B. Wang, B. Li, B. Dong, B. Zhao, C. Y. Li, Macromolecules 2010, 43, 9234.

[63] B. Li, C. Y. Li, J. Am. Chem. Soc. 2007, 129, 12.

[64] M. J. Hostetler, A. C. Templeton, R. W. Murray, Langmuir 1999, 15, 3782

[65] S. Imabayashi, D. Hobara, T. Kakiuchi, Langmuir 2001, 17, 2560.

[66] P. Ionita, A. Volkov, G. Jeschke, V. Chechik, Anal. Chem. 2008, 80, 95.

[67] Y. Song, L. M. Klivansky, Y. Liu, S. W. Chen, Langmuir 2011, 27, 14581. 